\title{
Evaluation of the Effect of Aerobic Exercise and Curcumin Consumption on HPG Axis (Hypothalamus-Pituitary-Gonadotropic) in Alcohol Binge Drinking Rats
}

\author{
Sara Heidarzadeh ${ }^{1}$, Mohammad Ali azarbayjani ${ }^{*}{ }^{1}$, Hassan Matin Homaee ${ }^{1}$, Mehdi Hedayati ${ }^{2}$ \\ 1- Department of Exercise Physiology, Central Tehran Branch, Islamic Azad University, Tehran, Iran \\ 2- Endocrine Research Center, Shaheed Beheshti University of Medical Sciences, Tehran, Iran
}

\section{A B S T R A C T}

Background and Objectives: Alcohol consumption has many health side effects. It is well known male gender is a serious risk factor to be an alcohol consumption and finding a way to reduce these complications in the short term is essential. The aim of this study was to investigate the interaction effects of aerobic exercise and curcumin on LH, FSH, Ts and GnRH following alcohol-induced rats.

Materials and Methods: 30 adult male Sprague Dawley rats with weighting 220-250 g and 8 weeks of age were obtained for this study, and randomly assigned into five groups $(\mathrm{n}=6)$ including: Dextrose-Saline control (Dext-Con), ethanol-control (Eth-Con), ethanol-curcumin (Eth-Cur), ethanol-swimming training (Eth-SWT) and ethanolSWT+curcumin (Eth-SWT+Cur). The project duration consisted of 4 days of addiction, 6 days of quitting, 14 days of swimming training (60 min/day) and curcumin $(50 \mathrm{mg} / \mathrm{kg})$ interventions, and finally animal sacrificed. Blood sample was collected and LH, FSH, Ts, and GnRH level were measured by using ELISA (enzyme-linked immunosorbent assay) kit. Analysis of variance (ANOVA) was performed using SPSS version 21.

Results: In the Eth-Con group, alcohol reduced the level of LH, FSH, Ts, and GnRH compared to other specially the Dext-Con group $(\mathrm{p}=0.001)$. In the Eth-SWT+Cur group, significantly increased of these hormones was observed $(\mathrm{p}=0.001)$. Exercise alone had no significantly effect on FSH and GnRH level.

Conclusions: Likely curcumin along exercise could improve HPG axis biomarker after a decline due to excessive alcohol consumption in rat. Lack of exercise effect alone can be due to Exercise-induced oxidative stress.

Keywords: Alcohol drinking, HPG axis, LH, FSH, GnRH, Testosterone, Aerobic exercise, Curcumin

\section{Introduction}

Alcohol addiction is described by a degree of clinical heterogeneity, that based on a greater diversity of the involved neurocircuitries and neuronal systems (1). Ethanol is one of the most widespread used recreational drinks $(2,3)$. Therefore, sexual dimorphisms in alcohol degradation are important when attempting to explain the differences of gender in alcohol addiction (4). Alcohol and its metabolite acetaldehyde have been classified as a group one carcinogens by several studies on different disorders $(2,3)$. Acute and chronic alcohol misuse and associated harmful effects have been investigated widely in causing damage to different organs and functions especially in reproductive function disorders in humans and experimental animals $(4,5)$. A direct association of alcohol addiction with metabolic functions and other hormones involved in reproduction at the gonadal level of the hypothalamus-pituitary-gonadotropic (HPG) axis is well established (4).

The HPG axis is a critical part in the reproductive and immune systems development and body's systems regulation that any alteration in this axis may cause hormones produced fluctuation or various systemic effects on the body. The axis controls reproduction through Gonadotropin-releasing hormone $(\mathrm{GnRH})$ to make luteinizing hormone $(\mathrm{LH})$, follicle-stimulating hormone (FSH), oestrogen and testosterone. This 
HPG axis is a multiple level hormonal system which involving with brain or pituitary with feed forward and feedback elements. Fundamentally, one of the mechanisms that medications or drugs may damage male fertility is the altering the HPG axis. High alcohol drinking or alcoholism cause alterations in the pituitary-gonadotropic (PG) axis, resulted in testicular dysfunction (4). Moreover, alcohol oxidation competes with the production of testicular and testosterone. These mechanisms leading to reduce in sperm density and the volume of semen (6). There are still no unified results in this area. Some researchers believe that alcohol act via the HPG axis to vary the pituitary gonadotropic hormone secretions $(5,7)$, while other authors stated that it acts directly on testicular tissue (8).

Nowadays, numerous substances change the delicately balanced HPG axis, such as dietary phytochemicals or natural plants could causing an alteration in intratesticular testosterone concentrations or a reduction in pituitary-secreted gonadotropins (9). The main constituent of the spice turmeric is Curcumin, which is a polyphenolic phytochemical with antioxidant, anti-inflammatory, anticancer, antiseptic, and hypocholesterolemic properties, which is effective in reducing the complications of diseases especially testicular dysfunction (10-15). In addition, Curcumin consumption improved alcohol-mediated fetal cardiac apoptosis, suggesting that curcumin play a protective role against alcohol abuse caused cardiac damage during pregnancy (15-18). Moreover, curcumin was also known to hinders the conversion of alcohol to acetaldehyde in testicular microsomal fractions (19).

Briefly, alcohol can damage the performance of the HPG axis $(4,19)$. In contrast, regular physical activity and consumption of curcumin alone have reduced the complications or decreased dysfunction of the HPG axis $(15,17,18,20-22)$. However, few studies have examined the simultaneous effect of these two variables $(22,23)$. The purpose of this study was to reduce the effects of excessive alcohol consumption in a short period. It is imperative to investigate that at least time and low doses of interventions reduce alcohol-related negative complications. Moreover, regular exercise leads to the adaptation in antioxidant capacity, protecting cells against harmful effects of oxidative stress (24). The relationship between antioxidant effects and HPG function improvement has been observed in male rats and female mice $(15$, 18). The synergistic effect of exercise and natural medications are widely proven to cure various illnesses (25). Therefore the aim of this study was to investigate the simultaneous effects of short-term aerobic and turmeric training on HPG axis biomarkers including of the level of LH, FSH, Ts, and GnRH (As the most important activity-based biomarkers of HPG axis) in rats.

\section{Materials and Methods}

\section{Experimental animals and ethical aspects}

In present study, 30 adult male Sprague-Dawley rats $(220-250 \mathrm{~g})$ in weight, and 8 weeks of age were obtained from the animal centre house of Islamic Azad University, Central Tehran Branch. The animals transferred to the laboratory polycarbonate cages as separate groups. They were reared at a temperature of $22 \pm 2{ }^{\circ} \mathrm{C}$ and $55 \pm 5 \%$ moisture under $12 / 12 \mathrm{~h}$ light/dark cycle. The day cycle began on 7.00 a.m. The capable rats of swimming exercise were randomly assigned into five groups $(n=6)$ : DextroseSaline control (Dext-Con, received a mixed of dextrose and Saline), ethanol-control (Eth-Con), ethanol-curcumin (Eth-Cur), ethanol-swimming training (Eth-SWT) and ethanol-SWT+curcumin (Eth-SWT+Cur). All procedures involving animal experiments were approved and carried out in strict accordance with the research guidelines for the care and use of laboratory animals by the Animal Care and Use Committee (ACUC).

\section{Binge ethanol induction}

The entire project duration consisted of 4 days of alcohol gavage (25\% ethanol w/v in vanilla Ensure TM; Abbot Laboratories, Columbus, $\mathrm{OH})(22,23,26)$, 6 days of quitting, 14 days of exercise and curcumin interventions, and finally animal sacrificed 48 hours after last exercise session. Access to food was restricted in all groups to control isocaloric diet during alcohol gavage. However, the calorie equivalent of alcohol was used in the healthy glucose group. The experiential groups were received Ethanol according to previous methods $(22,23,26)$. The initial dose of alcohol was administered $5 \mathrm{~g} / \mathrm{kg}$ of body weight, and then subsequent doses were administered depending on the behaviour of ethanol up to a maximum of $7 \mathrm{~g} / \mathrm{kg}$ of the body weight per day. After ethanol induction, the diet converted to the 
standard of animals consumed pellet food (Behparvar Co., Karaj, Iran).

\section{Curcumin supplement preparation}

Curcumin (1,7-bis[4-hydroxy-3-methoxyphenyl]1,6-heptadiene-3,5-dione) was purchased from Merck Company (Merck, 820354) and mixed with $10 \mathrm{mg} / \mathrm{ml}$ into curcumin solvent (Dimethyl sulfoxide: DMSO, Merck, 109678). The solution was intraperitoneally injected for 2 weeks (five times per week, the same as swimming sessions) with the dose of $50 \mathrm{mg} / \mathrm{kg}$ body weight.

\section{Swimming training protocol}

Swimming training was considered in this study. The training protocol was performed by using a swimming tank (Razi Rad Co. 0.5 m_0.45 m_0.45 m) with temperature of $20-22^{\circ} \mathrm{C}$. The experimental groups were conducted swimming training with each session of $60 \mathrm{~min} /$ day for the period of 2 weeks.

\section{Blood sampling and determination of biochemical variables}

Animals from all groups were euthanized with ketamine/xylazine ( $80 / 8 \mathrm{mg} / \mathrm{kg}$ i.p.) and sacrificed by cervical dislocation at the end of procedure. Blood samples were collected through a heart puncture to evaluate the biochemical variables. In this study, LH, FSH, Ts, and GnRH level were measured by using ELISA (enzyme-linked immunosorbent assay) kit.

\section{Statistical Analysis}

All data are presented as mean \pm standard deviation. Shapiro-Wilk test was conducted to determine the data are normally distribution. Analysis of variance (ANOVA) was performed using SPSS version 21. Two-way ANOVA was used for comparing the effect of exercise and Curcumin and the combination of them on biochemical parameters. A Tukey post-hoc analysis was used to heck for significant differences among the main effects of each dependent variable. Statistical significance was considered when $\mathrm{p}$ value was less than 0.05 .

\section{Results}

Alcohol consumption reduced the level of $\mathrm{LH}$ compared to the other groups especially Dex-Con group. The exercise and curcumin consumption significantly increased the concentration of $\mathrm{LH}$ compared to the ethanol groups $(\mathrm{F}=472.21, \mathrm{P}=0.001$, $\eta=0.938),(\mathrm{F}=511.27, \mathrm{P}=0.001, \mathrm{\eta}=0.905)$ respectively. Moreover, the elevation of $\mathrm{LH}$ was more pronounced in the group of interaction of exercise and curcumin compared to the other ethanol groups $(\mathrm{F}=379.64$, $\mathrm{P}=0.001, \mathrm{\eta}=0.954$ ) (Figure-1).

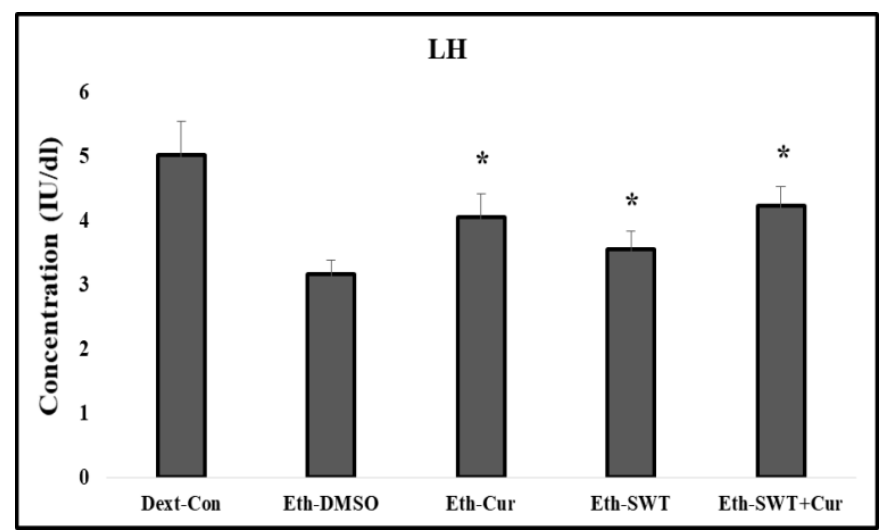

Figure 1. The concentration of LH among studied groups. Dext-Con; Dextrose-Saline control, Eth-DMSO; ethanolDMSO, Eth-Cur; ethanol-curcumin, Eth-SWT; ethanol + swimming training, Eth-SWT + Cur ethanol + swimming training + curcumin. ${ }^{*} \mathrm{p}<0.05$ as compared to Dext-Con.

The level of FSH is reduced through alcohol consumption compared to the other groups especially Dex-Con group. Curcumin consumption significantly increased the concentration of FSH compared to the Eth-Con group $(\mathrm{F}=598.46, \mathrm{P}=0.001, \mathrm{\eta}=0.875)$. The exercise alone had no significantly effect on FSH level ( $\mathrm{F}=638.41, \mathrm{P}=0.036, \mathrm{\eta}=0.095$ ). Furthermore, the level of FSH also significantly increased in the group of interaction of exercise and curcumin consumption compared to the other ethanol groups $(\mathrm{F}=379.36$, $\mathrm{P}=0.001, \eta=0.954$ ) (Figure-2).

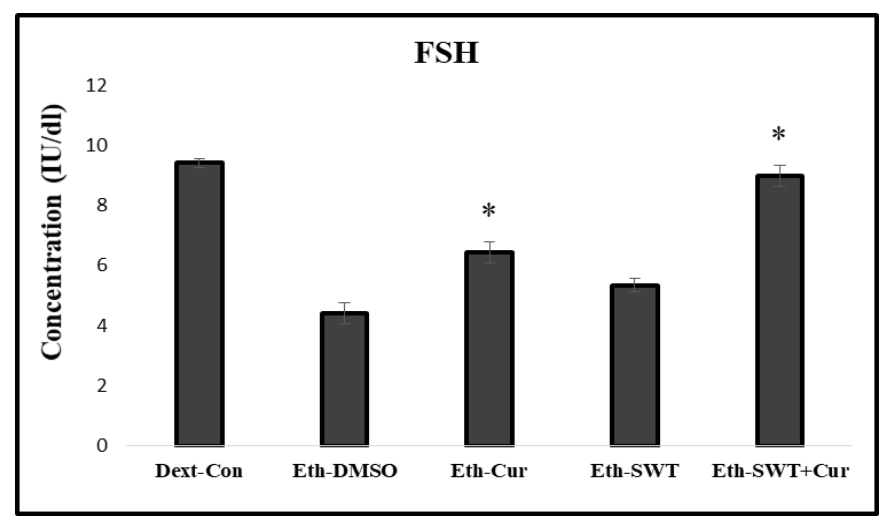

Figure 2. The concentration of FSH among studied groups. Dext-Con; Dextrose-Saline control, Eth-DMSO; ethanolDMSO, Eth-Cur; ethanol-curcumin, Eth-SWT; ethanol + swimming training, Eth-SWT + Cur ethanol + swimming training + curcumin. $* \mathrm{p}<0.05$ as compared to Dext-Con.

Alcohol consumption reduced the level of testosterone compared to the other groups especially Dex-Con group. The exercise $(\mathrm{F}=448.15, \mathrm{P}=0.001$, $\eta=0.973)$, and Curcumin $(\mathrm{F}=398.26, \quad \mathrm{P}=0.001$, 
$\eta=0.972$ ) increased the concentration of testosterone compared to the other ethanol groups. Additionally, the interaction of exercise and curcumin was more pronounced on testosterone level improved compared to the other ethanol groups $(\mathrm{F}=387.34, \mathrm{P}=0.001$, $\eta=0.954$ )(Figure-3).

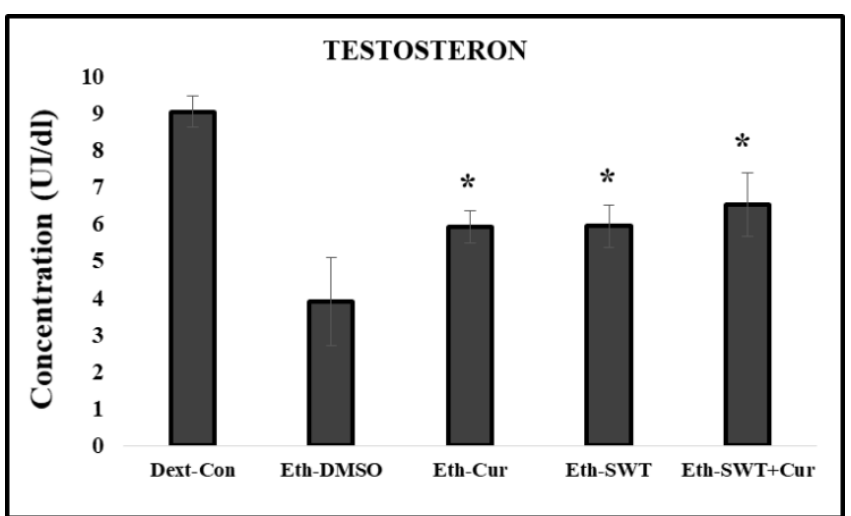

Figure 3. The concentration of Testosterone among studied groups. Dext-Con; Dextrose-Saline control, Eth-DMSO; ethanol-DMSO, Eth-Cur; ethanol-curcumin, Eth-SWT; ethanol + swimming training, Eth-SWT + Cur ethanol + swimming training + curcumin. ${ }^{*} \mathrm{p}<0.05$ as compared to Dext-Con.

The level of $\mathrm{GnRH}$ is reduced through alcohol consumption compared to the other groups especially Dex-Con group. Curcumin consumption significantly increased the concentration of $\mathrm{GnRH}(\mathrm{F}=525.36$, $\mathrm{P}=0.001, \eta=0.904)$ compared to the other ethanol groups. The exercise alone had no significantly effect on GnRH level compared to the other ethanol groups $(\mathrm{F}=652.39, \mathrm{P}=0.059, \mathrm{\eta}=0.165)$. Furthermore, the level of GnRH also significantly increased in the group of interaction of exercise and Curcumin consumption compared to the other ethanol groups $(\mathrm{F}=417.22$, $\mathrm{P}=0.001, \mathrm{\eta}=0.971$ )(Figure-4).

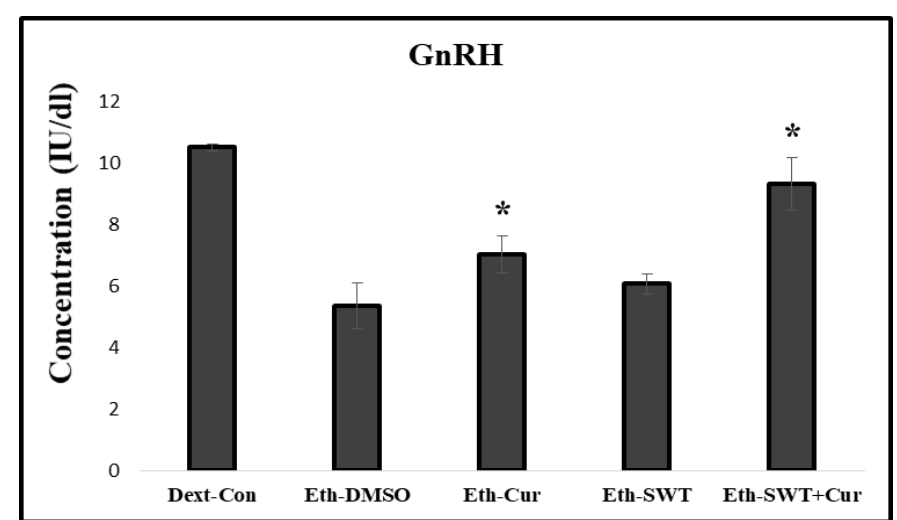

Figure 4. The concentration of GnRH among studied groups. Dext-Con; Dextrose-Saline control, Eth-DMSO; ethanol-DMSO, Eth-Cur; ethanol-curcumin, Eth-SWT; ethanol + swimming training, Eth-SWT + Cur ethanol + swimming training + curcumin. $* \mathrm{p}<0.05$ as compared to Dext-Con.

\section{Discussion}

The findings of present study clearly demonstrate that LH, FSH, testosterone, and GnRH level decreased in rats who addiction with alcohol and a period of 2 weeks swimming training and Curcumin consumption had significantly effect on the improving of these factors. Based on the changes observed in the present study, it can be stated that excessive alcohol consumption affects the performance of the HPG axis. Exercise alone reduces these effects to some extent. Curcumin alone produces a better effect than exercise. The interactive effect of both independent variables led to further improvement. It can be noted that exercise has less effect for two reasons. First, high intensity exercise increases oxidative stress. Another reason was the shortness of training. It is likely that increasing the workout period can produce better effects through better adaptation. It also increases the intensity of exercise led to increase lactic acid, and lactic acid increases testosterone secretion from testis (27). This may decrease secretion of other biomarkers in the exercise group alone by negative feedback compared to the curcumin interaction group. However, the interaction of exercise and curcumin with the antioxidant properties of turmeric may counteract the exercise-induced oxidative stress.

It is well established that alcohol drinking inhibits testosterone production and probably causes testicular dysfunction (4). Because the HPG axis are steroid hormones, this change can affect other organs of the body. The early sex hormone activity model of alcohol addiction may prove to be a valuable tool in the development of preventive and therapeutic strategies (1), as the levels of corticosterone was increased in male rats which consumed alcohol (28). The mechanisms underlying the fertility damage by alcohol are not yet fully clarified. Furthermore, it is well stablished that curcumin protect damaged organs affected by alcohol consumption (18, 29-31). The results of present study may portend that alcohol acts on HPG axis biomarkers dysfunction through both pathways via disruption of the hypothalamuspituitary-gonadal axis and directly on the testis activity biomarkers after two weeks of aerobic exercise and curcumin consumption. This study also shows that the administration of alcohol reduced the level of LH, however exercise and curcumin consumption significantly increased the concentration of $\mathrm{LH}$, but the elevation of $\mathrm{LH}$ was more pronounced 
in the group that performed exercise and received curcumin. These findings are in consonant with reports from Ren et al. (32) and Oremosu et al.(33). Some authors who report that the negative feedback of testosterone on the hypothalamus-pituitarygonadal (HPG) axis promotes an increase in LH (8, 34, 35). Recent evidence suggests that due to the intensity of exercise by increasing lactic acid and its effect on HPG axis, testicular gland to increase testosterone secretion improve semen quality, hormone values, and male fertility $(20,21,27,36$, 37). Curcumin shows to have efficacious protection against alcohol-induced tissue damage (17, 30). Our LH finding shows that the animals that were treated with alcohol had low LH levels, but after 2 weeks of exercise and consuming Curcumin, the LH level reached to acceptable level. It means that there is an interaction of two variables to have synergistic effect on the level of LH hormone.

The results of present study indicated that the level of FSH is reduced via alcohol consumption, however, Curcumin significantly increased the concentration of FSH, but exercise alone had no effect on FSH level. Furthermore, the level of FSH also significantly increased in the group of exercise and Curcumin. Exercise training alone had no effect on FSH level, that may associated to the intensity of exercise or needs to perform longer than two weeks to be effective. It has been shown that alcohol could decline the production of LH and FSH through influencing on the anterior pituitary gland (32). Contrary, it has been demonstrated that chronic alcoholics had a significant increased on FSH and LH levels (8). The results of alcohol on HPG axis pathway are controversy that may be related to research condition or other factors, which can effect on this axis. Furthermore, alcohol consumption not only able to reduce LH and FSH synthesis but can prevent the secretion of these hormones. Curcumin has antioxidant properties and oral administration of curcumin with alcohol showed a significant reduction in negative alcohol effects side in mic model (29). Aerobic exercise alongside Curcumin consumption seems to improve alcohol effects.

In the present study, a significantly decrease was observed in the level of testosterone within alcohol consumption, however, exercise and Curcumin increased the concentration of testosterone. In addition, the interaction of exercise and Curcumin was more effective on testosterone level improved. This hormone is essential for spermatogenesis which reduced in alcohol binge drinking rats in agreement with previous reports (8). Numerous constituents vary the delicate balance of HPG axis, that causes either a reduction in pituitary-secrete gonadotropins or a modification of testosterone concentrations (9). Alcohol has a direct toxic effect on the testis that leads to decreased seminiferous tubular function (30). A study has proven that the significant reduction of sperm motility and sperm count in animals with alcohol treated resulted in inimical to male fertility (33). Curcumin suppress the conversion of alcohol to acetaldehyde in testicular microsomal fractions (19). The effectiveness of exercise and Curcumin consumption are in increasing testosterone levels explained may directly effect of HPG axis and GnRH release from the hypothalamus.

$\mathrm{GnRH}$ is the important key regulator of the reproductive axis. The level of GnRH in the present study is reduced through alcohol consumption, while curcumin significantly increased the concentration of GnRH, and curcumin alongside exercise treatment increased GnRH. It has been shown that testosterone levels modify HPG axis, and high level of this hormone suppresses the fluctuation of $\mathrm{GnRH}$ pulsatile release from the hypothalamus (38). Our data indicated that exercise alone had no markedly effect on inducing elevation GnRH level, which can be due to the intensity of exercise and exercise-induced oxidative stress or the short term of our selected protocol and low adaptation. In matching with our results, regular moderate exercise treatment had no effects on the GnRH level (39). In contrast, a study has proven that moderate exercise improve the performance of the pituitary-gonadal axis and fertility (40). Thus, the results confirm that Curcumin with exercise have a synergistic impact on the HPG axis biomarkers that will probably improve fertility performance. Note that these findings have been reported in male rats and should be generalized.

\section{Conclusion}

Reduction of the HPG axis activity under the influence of each factor in particular alcohol can decrease the hormone biomarkers of this axis especially LH and FSH and may leads to decrease spermatogenesis and fertility function consequently. To sum up, the findings of the present study indicated that likely consumption of the curcumin combined 
with aerobic exercise is a therapeutic effective factor to reverse the HPG axis damage via alcohol effect invivo. Notice that in the present study, smaller groups and shorter periods were used to minimize injury to animals. Therefore, this may affect the results. Future studies should also document the findings of gene expression and histology.

\section{Financial disclosure}

The authors declared no financial interest.

\section{Funding/Support}

There are no funding.

\section{References}

1. Lenz B, Müller CP, Stoessel C, Sperling W, Biermann $\mathrm{T}$, Hillemacher $\mathrm{T}$, et al. Sex hormone activity in alcohol addiction: integrating organizational and activational effects. Progress in Neurobiology. 2012;96(1):136-63.

2. Peacock A, Leung J, Larney S, Colledge S, Hickman M, Rehm J, et al. Global statistics on alcohol, tobacco and illicit drug use: 2017 status report. Addiction. 2018;113(10):1905-26.

3. Soler-Vila H, Ortolá R, García-Esquinas E, LeónMuñoz LM, Rodríguez-Artalejo F. Changes in Alcohol Consumption and Associated Variables among Older Adults in Spain: A population-based cohort study. Scientific Reports. 2019;9(1):10401.

4. Lenz B, Muller CP, Stoessel C, Sperling W, Biermann $\mathrm{T}$, Hillemacher T, et al. Sex hormone activity in alcohol addiction: integrating organizational and activational effects. Prog Neurobiol. 2012;96(1):136-63.

5. Frias J, Torres JM, Miranda MT, Ruiz E, Ortega E. Effects of acute alcohol intoxication on pituitarygonadal axis hormones, pituitary-adrenal axis hormones, beta-endorphin and prolactin in human adults of both sexes. Alcohol and alcoholism (Oxford, Oxfordshire). 2002;37(2):169-73.

6. Purohit V. Can alcohol promote aromatization of androgens to estrogens? A review. Alcohol. 2000;22(3):123-7.

7. Ren J-C, Banan A, Keshavarzian A, Zhu Q, LaPaglia $\mathrm{N}$, McNulty $\mathrm{J}$, et al. Exposure to ethanol induces oxidative damage in the pituitary gland. Alcohol. 2005;35(2):91-101.

8. Muthusami K, Chinnaswamy P. Effect of chronic alcoholism on male fertility hormones and semen quality. Fertility and sterility. 2005;84(4):919-24.

9. Weber R, Pierik F, Dohle G, Burdorf A. Environmental influences on male reproduction. BJU international. 2002;89(2):143-8.

10. Duvoix A, Blasius R, Delhalle S, Schnekenburger M, Morceau F, Henry E, et al. Chemopreventive and therapeutic effects of curcumin. Cancer letters. 2005;223(2):181-90.
11. Maheshwari RK, Singh AK, Gaddipati J, Srimal RC. Multiple biological activities of curcumin: a short review. Life sciences. 2006;78(18):2081-7.

12. Priyadarsini KI, Maity DK, Naik G, Kumar MS, Unnikrishnan M, Satav J, et al. Role of phenolic $\mathrm{OH}$ and methylene hydrogen on the free radical reactions and antioxidant activity of curcumin. Free Radical Biology and Medicine. 2003;35(5):475-84.

13. Suryanarayana P, Satyanarayana A, Balakrishna N, Kumar PU, Reddy GB. Effect of turmeric and curcumin on oxidative stress and antioxidant enzymes in streptozotocin-induced diabetic rat. Medical Science Monitor. 2007;13(12):BR286-BR92.

14. Sun H, Zhu J, Lu T, Huang X, Tian J. Curcuminmediated cardiac defects in mouse is associated with a reduced histone $\mathrm{H} 3$ acetylation and reduced expression of cardiac transcription factors. Cardiovascular toxicology. 2014;14(2):162-9.

15. Akintunde JK, Farouk AA, Mogbojuri O. Metabolic treatment of syndrome linked with Parkinson's disease and hypothalamus pituitary gonadal hormones by turmeric curcumin in Bisphenol-A induced neurotesticular dysfunction of wistar rat. Biochemistry and biophysics reports. 2019;17:97-107.

16. Yan X, Pan B, Lv T, Liu L, Zhu J, Shen W, et al. Inhibition of histone acetylation by curcumin reduces alcohol-induced fetal cardiac apoptosis. Journal of biomedical science. 2017;24(1):1.

17. Mahmoudi R, Honarmand Z, Karbalay-Doust S, JafariBarmak M, Nikseresht $M$, Noorafshan A. Using curcumin to prevent structural impairments of testicles in rats induced by sodium metabisulfite. EXCLI journal. 2017;16:583-92.

18. Yan Z, Dai Y, Fu H, Zheng Y, Bao D, Yin Y, et al. Curcumin exerts a protective effect against premature ovarian failure in mice. Journal of molecular endocrinology. 2018;60(3):261-71.

19. Quintans LN, Castro GD, Castro JA. Oxidation of ethanol to acetaldehyde and free radicals by rat testicular microsomes. Archives of toxicology. 2005;79(1):25-30.

20. Azarbayjani MA, Fatolahi H, Rasaee MJ, Peeri M, Babaei R. The effect of exercise mode and intensity of sub-maximal physical activities on salivary testosterone to cortisol ratio and alpha-amylase in young active males. International journal of exercise science. 2011;4(4):283-93.

21. Dehghan F, Khodaei F, Afshar L, Shojaei FK, Poorhakimi E, Soori R, et al. Effect of competition on stress salivary biomarkers in elite and amateur female adolescent inline skaters. Science \& Sports. 2019;34(1):e37-e44.

22. Fatolahi H, Azarbayjani MA, Peeri M, Matinhomaei H. The effect of curcumin and exercise rehabilitation on liver paraoxonase-1 and NF-kbeta gene expression in 
the rat induced by forced drinking of ethanol. Clinical and experimental hepatology. 2020;6(1):49-54.

23. fatolahi h, Azarbayjani MA, Peeri M, Mateen Homaei $\mathrm{H}$. The effect of short-term training and curcumin on the paraoxonase- 1 activity after alcohol withdrawal in male Wistar rats. EBNESINA. 2019;20(4):11-8.

24. Ciolac EG, Guimarães GV. Physical exercise and metabolic syndrome. Rev Bras Med Esporte. 2004;10:319-24.

25. Khosravani M, Azarbayjani MA, Abolmaesoomi M, Yusof A, Zainal Abidin N, Rahimi E, et al. Ginger extract and aerobic training reduces lipid profile in high-fat fed diet rats. European review for medical and pharmacological sciences. 2016;20(8):1617-22.

26. Maynard ME, Leasure JL. Exercise enhances hippocampal recovery following binge ethanol exposure. PloS one. 2013;8(9):e76644.

27. Lu SS, Lau CP, Tung YF, Huang SW, Chen YH, Shih $\mathrm{HC}$, et al. Lactate and the effects of exercise on testosterone secretion: evidence for the involvement of a cAMP-mediated mechanism. Medicine and science in sports and exercise. 1997;29(8):1048-54.

28. Fahlke C, Engel JA, Eriksson CP, Härd E, Söderpalm B. Involvement of corticosterone in the modulation of ethanol consumption in the rat. Alcohol. 1994;11(3):195-202.

29. Giannessi F, Giambelluca MA, Grasso L, Scavuzzo MC, Ruffoli R. Curcumin protects Leydig cells of mice from damage induced by chronic alcohol administration. Medical Science Monitor. 2008;14(11):BR237-BR42.

30. Sudjarwo SA, Sudjarwo GW, Koerniasari. Protective effect of curcumin on lead acetate-induced testicular toxicity in Wistar rats. Research in pharmaceutical sciences. 2017;12(5):381-90.

31. Wang Q, Wang J, Sun LJ, Hu LP, Li J, Shao JQ, et al. [Salidroside protects the hypothalamic-pituitary-gonad axis of male rats undergoing negative psychological stress in experimental navigation and intensive exercise]. Zhonghua nan ke xue $=$ National journal of andrology. 2009;15(4):331-6.
32. Ren JC, Banan A, Keshavarzian A, Zhu Q, Lapaglia N, McNulty J, et al. Exposure to ethanol induces oxidative damage in the pituitary gland. Alcohol. 2005;35(2):91101.

33. Oremosu AA, Akang EN. Impact of alcohol on male reproductive hormones, oxidative stress and semen parameters in Sprague-Dawley rats. Middle East Fertility Society Journal. 2015;20(2):114-8.

34. Shetty KT, Ray R, Sengupta SN, Desai NG. PITUITARY GONADAL FUNCTIONING IN MALE ALCOHOLICS IN AN INDIAN PSYCHIATRIC HOSPITAL. Alcohol and Alcoholism. 1991;26(1):4751.

35. Heinz A, Rommelspacher H, Gräf K-J, Kürten I, Otto M, Baumgartner A. Hypothalamic-pituitary-gonadal axis, prolactin, and cortisol in alcoholics during withdrawal and after three weeks of abstinence: comparison with healthy control subjects. Psychiatry Research. 1995;56(1):81-95.

36. Vaamonde D, Da Silva-Grigoletto ME, Garcia-Manso JM, Barrera N, Vaamonde-Lemos R. Physically active men show better semen parameters and hormone values than sedentary men. European journal of applied physiology. 2012;112(9):3267-73.

37. Gaskins AJ, Mendiola J, Afeiche M, Jorgensen N, Swan SH, Chavarro JE. Physical activity and television watching in relation to semen quality in young men. British journal of sports medicine. 2015;49(4):265-70.

38. Bagatell CJ, Bremner WJ. Androgens in men-uses and abuses. New England Journal of Medicine. 1996;334(11):707-15

39. Khajehnasiri N, Khazali H, Sheikhzadeh F. Various responses of male pituitary-gonadal axis to different intensities of long-term exercise: Role of expression of KNDYrelated genes. Journal of biosciences. 2018;43(4):569-74.

40. Sharma R, Biedenharn KR, Fedor JM, Agarwal A. Lifestyle factors and reproductive health: taking control of your fertility. Reproductive Biology and Endocrinology. 2013;11(1):66. 